\title{
Micro-macro transition and simplified contact models for wet granular materials
}

\author{
Sudeshna Roy $^{1}$ - Abhinendra Singh ${ }^{1} \cdot$ Stefan Luding $^{1} \cdot$ Thomas Weinhart $^{1}$
}

Received: 1 May 2015 / Accepted: 21 July 2015 / Published online: 7 August 2015

(C) The Author(s) 2015. This article is published with open access at Springerlink.com

\begin{abstract}
Wet granular materials in a quasistatic steadystate shear flow have been studied with discrete particle simulations. Macroscopic quantities, consistent with the conservation laws of continuum theory, are obtained by time averaging and spatial coarse graining. Initial studies involve understanding the effect of liquid content and liquid properties like the surface tension on the macroscopic quantities. Two parameters of the liquid bridge contact model have been identified as the constitutive parameters that influence the macroscopic rheology (i) the rupture distance of the liquid bridge model, which is proportional to the liquid content, and (ii) the maximum adhesive force, as controlled by the surface tension of the liquid. Subsequently, a correlation is developed between these microparameters and the steady-state cohesion in the limit of zero confining pressure. Furthermore, as second result, the macroscopic torque measured at the walls, which is an experimentally accessible parameter, is predicted from our simulation results with the same dependence on the microparameters. Finally, the steady- state cohesion of a realistic non-linear liquid bridge contact model scales well with the steady-state cohesion for a simpler linearized irreversible contact model with the same maximum adhesive force and equal energy dissipated per contact.
\end{abstract}

Sudeshna Roy

s.roy@utwente.nl

Abhinendra Singh

a.singh@utwente.nl

Stefan Luding

s.luding@utwente.nl

Thomas Weinhart

t.weinhart@utwente.nl

1 Multi Scale Mechanics (MSM), Engineering Technology (CTW) and MESA+, University of Twente, P.O. Box 217, 7500 AE Enschede, The Netherlands
Keywords Rheology · Wet granular materials - DEM · Micro-macro transition $\cdot$ Cohesion

\section{Introduction}

Granular media are collections of microscopic grains having athermal interactions through dissipative, frictional, or cohesive contact forces. External force leads to granular flow under the condition of applied shear stress exceeding the yield shear stress. After a finite shear strain, at constant rate, a steady state establishes with a typically lower shear stress, depending on both strain rate and pressure [1]. Most studies in granular physics focus on dry granular materials and their flow rheology. However, wet granular materials are ubiquitous in geology and many real-world applications, where interstitial liquid is present between the grains. Simplified models for capillary clusters [2,3] and wet granular gases [4] were introduced before. The rheology of flow for dense suspension of non-Brownian particles have been studied in Refs. [5-7]. We study the local rheology of weakly wetted granular materials in the quasistatic regime with the discrete element method (DEM) using the open-source package MercuryDPM $[8,9]$ in a shear cell set-up, where the relative motion is confined to particles in a narrow region away from the walls, called shear band $[10,11]$. We study partially saturated systems in the pendular regime, with a very low level of water content, where the formation of liquid bridges between particle pairs leads to development of microscopic tensile forces. Other forces such as the electrostatic double layer forces can occur between charged objects across liquids, typically dipolar as water. These forces are most active in systems with high surface area to volume ratio, such as colloids or porous materials. We neglect the effect of such forces in our system of rather large $(\sim \mathrm{mm})$ non-porous glass 
particles. The tensile forces generated at particle level result in cohesion at macroscopic scale. Earlier studies have been done for liquid bridge in the pendular regime to understand the effect of liquid bridge volume and contact angle on different macroscopic quantities like the steady-state cohesion, torque, and shear band properties [12-16]. Other studies for unsaturated granular media observe fluid depletion in shear bands $[17,18]$. However, there is no theoretical framework or concrete model available yet that defines the exact correlation between the microparameters like the liquid bridge volume and the surface tension of the liquid with the steady-state cohesion.

The liquid bridge contact model is based on the experimental study of [19] where the capillary force was obtained by measuring the apparent weight of a moving upper sphere by a sensitive microbalance. The lower sphere was attached to a piezoelectric actuator which controlled the separation between the two surfaces. The distance between the two solid surfaces of the spheres was obtained from the position of the piezo-actuator. In order to develop a micro-macro correlation for the liquid bridge contact model, we initially study the structure of the microcontact model. How is the structure of the liquid bridge contact model affected by the microscopic parameters? How does this influence the steady-state cohesion? Here we study in detail on the effect of these parameters on the macro-results. For example, the effect of maximum interaction distance, or the distance at which the liquid bridge between two interacting particles ruptures, is studied by varying the liquid content. On the other hand, other parameters like surface tension of the liquid and contact angle affect the magnitude of force acting between the particles when in contact $[14,19]$. Various surface tensions of liquids give a large scale variation of the capillary force and this allows us to study the effect of maximum force on the macroscopic properties. Furthermore, in the consecutive analysis, we re-obtain the macro-rheology results in the shear band center from the torque, torque being an experimentally measurable quantity.

The liquid bridge interactions between the particles are defined by the free-surface equilibrium shapes and stability of the bridge configuration between them [20-22]. Phenomenologically, even the simplified models of liquid bridges are quite complex in nature. In order to improve the computational efficiency for wet granular materials, we replace the non-linear interactions of liquid bridges with a simpler linear one. But in what way can a non-linear model like the liquid bridge contact model be replaced by a linear model? When can we say that the two different contact models are analogous? Therefore, we compare the realistic liquid bridge model with an equivalent simple linear irreversible contact model [23] that would give the same macroscopic effect.

The results in this paper are organized in three main parts. In Sect. 3.1 of this paper, we study the effect of varying liquid bridge volume and surface tension of the liquid on the macro- scopic properties, the focus being to find a micro-macro correlation from this study. Most strikingly, we see a welldefined relationship between these microparameters and the macroproperties like the steady-state cohesion of the bulk material and macro-torque required under shear, neglecting the effect of fluid depletion in shear bands $[17,18]$ in quasistatic flow. In Sect. 3.2 of this paper, we show the derivation of macro-torque from the boundary shear stress. In this section we also compare this torque with the torque calculated from forces due to contacts on the wall particles. In Sect. 4 of this paper, we discuss about the analogy of two different contact models, with a goal to understand which parameters at microscopic scale would give the same macroscopic behavior of the system.

\section{Model system}

\subsection{Geometry}

Split-bottom ring shear cell The set-up used for simulations consists of a shear cell with annular geometry and a split in the bottom plate, as shown in Fig. 1. Some of the earlier studies in similar rotating set-up include [24-26]. The geometry of the system consists of an outer cylinder (radius $R_{\mathrm{O}}=110 \mathrm{~mm}$ ) rotating around a fixed inner cylinder (radius $R_{\mathrm{i}}=14.7 \mathrm{~mm}$ ) with a rotation frequency of $f_{\text {rot }}=0.01 \mathrm{~s}^{-1}$. The granular material is confined by gravity between the two concentric cylinders, the bottom plate, and a free top surface. The bottom plate is split at radius $R_{\mathrm{S}}=85 \mathrm{~mm}$ into a moving outer part and a static inner part. Due to the split at the bottom, a shear band is formed at the bottom. It moves inwards and widens as it goes up, due to the geometry. This set-up thus features a wide shear band away from the wall, free from boundary effects, since an intermediate filling height $(H=40 \mathrm{~mm})$ is chosen, so that the shear band does not reach the inner wall at the free surface.

In earlier studies $[1,27,28]$, similar simulations were done using a quarter of the system $\left(0^{\circ} \leq \phi \leq 90^{\circ}\right)$ with periodic boundary conditions. In order to save computation time, here we simulate only a smaller section of the system $\left(0^{\circ} \leq\right.$ $\phi \leq 30^{\circ}$ ) with appropriate periodic boundary conditions in the angular coordinate, unless specified otherwise. We have observed no noticeable effect on the macroscopic behavior in comparisons between simulations done with a smaller $\left(30^{\circ}\right)$ and a larger $\left(90^{\circ}\right)$ opening angle. Note that for very strong attractive forces, the above statement is not true anymore.

\subsection{Microscopic model parameters}

In the presence of a small amount of liquid in a dense granular material, bridges are formed at the contact points between the particles. The surface energy of these bridges leads to an 




Fig. 1 Shear cell set-up

Table 1 Model parameters

\begin{tabular}{lll}
\hline Parameter & Symbol & Value \\
\hline Sliding friction coefficient & $\mu_{\mathrm{p}}$ & 0.01 \\
Elastic stiffness & $k$ & $120 \mathrm{~N} \mathrm{~m}^{-1}$ \\
Viscous damping coefficient & $\gamma_{\mathrm{o}}$ & $0.5 \times 10^{-3} \mathrm{~kg} \mathrm{~s}^{-1}$ \\
Angular frequency & $\omega$ & $0.01 \mathrm{~s}^{-1}$ \\
Particle density & $\rho$ & $2000 \mathrm{~kg} \mathrm{~m}^{-3}$ \\
Mean particle diameter & $d_{\mathrm{p}}$ & $2.2 \mathrm{~mm}$ \\
Contact angle & $\theta$ & $20^{\circ}$ \\
\hline
\end{tabular}

attractive force between the particles, which is absent in dry granular materials. Thus, wetting changes a granular system from one with only repulsive inter-particle interactions to one with both repulsive and attractive interactions [29]. With the change in microscopic physical interactions in wet granular materials, the macroscopic behavior is also expected to differ from the dry materials. Therefore, we choose to vary some of the characteristic specifications of a liquid bridge model to understand the effect on macroscopic properties. All the particle specifications and the fixed interaction parameters for the contact models are given in Table 1. All the variable interaction parameters which include the liquid bridge volume $V_{\mathrm{b}}$ and the surface tension of the liquid $\gamma$ are discussed in this section.

\subsubsection{Bulk saturation and liquid bridge volume}

The bulk material can be characterized by different states such as the dry bulk, adsorption layers, pendular state, funicular state, capillary state, or suspension depending on the level of saturation [30,31]. In this paper we intend to study the phenomenology of liquid bridge between particles in the pendular state, where the well-separated liquid bridges exist between particle pairs without geometrical overlap. In this section, we discuss about the critical bulk saturation of granular materials and the corresponding liquid bridge volumes in the pendular state.

The bulk saturation $S^{*}$ is defined as the ratio of liquid volume to void volume of the bulk [32-34]. The demarcation between the pendular state and the more saturated funicular state is given by the saturation $S^{*} \approx 0.3$ [32]. For each particle pair with a liquid bridge, a dimensionless volume $\varphi^{*}$ can be defined as the ratio of the volume of the liquid bridge at the contact, $V_{\mathrm{b}}$ to the volume of the two contacting particles, $2 V_{\mathrm{p}}$

$\varphi^{*}=\frac{V_{\mathrm{b}}}{2 V_{\mathrm{p}}}=\frac{V_{\mathrm{b}}}{2\left(\frac{\pi}{6} d_{\mathrm{p}}^{3}\right)}$.

Assuming the liquid is homogeneously distributed throughout the material, the bulk saturation $S^{*}$ is obtained from the dimensionless volume $\varphi^{*}$ and the bulk porosity $\epsilon$ from the following equation [32-34]:

$S^{*}=\pi \frac{1-\epsilon}{\epsilon^{2}} \varphi^{*}$.

With a bulk porosity of the material $\epsilon=0.4$ and a mean particle diameter $d_{\mathrm{p}}$ of $2.20 \mathrm{~mm}$, the maximum liquid bridge volume in the pendular regime is approximately $284 \mathrm{nl}$. In order to study the influence of liquid content on the macroscopic properties, we analyzed the system for the following set of liquid bridge volumes $V_{\mathrm{b}}$

$V_{\mathrm{b}} \in\{0,1,2,4.2,8,14,20,75,140,200\} \mathrm{nl}$,

which are seen to be well within the pendular regime. We also calculate the liquid volume as a percentage of the total volume of the system $\left(V_{\mathrm{t}}\right)$ based on the number of contacts. The number of contacts represented as $C_{\mathrm{L}}$ increases with increasing liquid bridge volume in the system and is measured approximately

$C_{\mathrm{L}} \in\{33010,36214,36855,37585,38306,39101$,

$$
39511,41526,42595,43328\} \text {. }
$$

Therefore, the volume percentage of liquid in the system is given by $\varphi_{\mathrm{b}}=C_{\mathrm{L}} V_{\mathrm{b}} / V_{\mathrm{t}}$ and is approximately equal to

$\varphi_{\mathrm{b}} \in\{0,0.03,0.07,0.15,0.29,0.52$,

$$
0.75,2.94,5.63,8.18\} \text {. }
$$

In order to investigate the functional form of steady-state cohesion beyond this state, a few more simulations for higher $V_{\mathrm{b}}$ are done: 
$V_{\mathrm{b}} \in\{500,1000\} \mathrm{nl}$,

for which the pendular assumption is not valid anymore.

\subsubsection{Surface tension of liquid}

Surface tension results from the greater attraction of liquid molecules toward each other than toward air. It is the tendency of liquids to lower their state of energy which makes it acquire the least possible surface area at the surface with higher interliquid molecules attraction. As a result, cohesive properties of liquids are reflected in surface tension which makes it an interesting parameter to study. This effect will be discussed in detail in Sect. 2.3.1. The effect of surface tension on the macroscopic properties is studied for the following range of surface tension values:

$\gamma \in\{0,0.020,0.040,0.060\} \mathrm{Nm}^{-1}$.

Surface tension of most of available liquid-air interfaces at $20{ }^{\circ} \mathrm{C}$ is in this range. To investigate the functional behavior of steady-state cohesion beyond this state, a few more simulations for higher $\gamma$ are done:

$\gamma \in\{0.01,0.10,0.50,1.00\} \mathrm{Nm}^{-1}$.

\subsection{Liquid bridge contact model}

The contact and non-contact forces for interacting particles can be described by a combination of an elastic contact model for the normal repulsive force and a non-linear irreversible adhesive model for the non-contact adhesive force. Figure 2 represents a sketch of the combined liquid bridge contact model as a function of the overlap between the two particles.

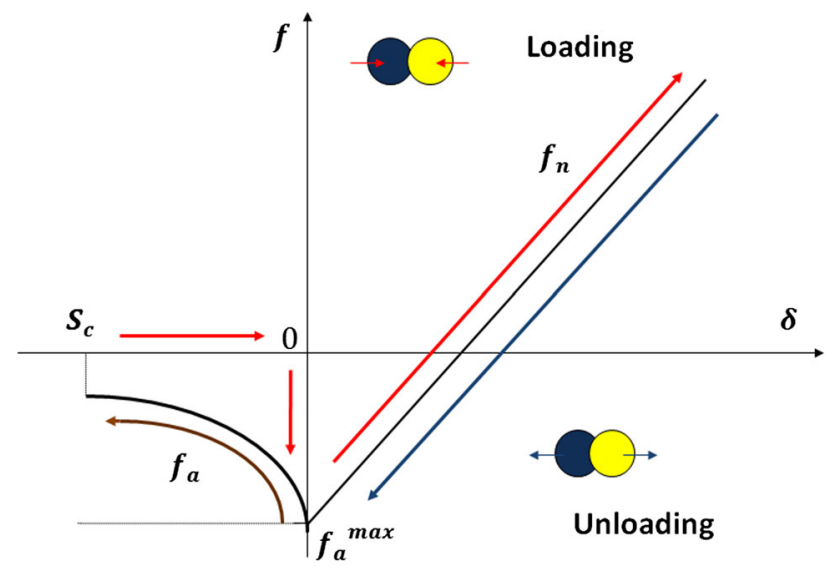

Fig. 2 Liquid capillary bridge model. The red lines represent the loading direction, the blue line represents the unloading direction when the particles are in contact and the brown line represents the unloading for the non-contact particles with short-range interaction force. (Color figure online)
The liquid bridge adhesive force acts between the particles once the contact is established and the liquid bridge is formed. According to the experimental measurements of [19], an increase in downward force is detected in the microbalance as soon as the liquid bridge is formed. Here, we assume that the liquid bridge formation and the contact establishment occur simultaneously and thus the capillary force becomes active during loading at first contact i.e., $\delta=0$. When the particles are in contact, the attractive force is given by Eq. (13). This is independent of the liquid bridge volume and depends on the surface tension of the liquid, radius of particles, and contact angle. There is no cohesive force between the particles during approach. As the liquid bridge only forms once the particles come in contact with each other, the cohesive force starts acting and remains constant during overlap between particles $\delta>0$. Normal contact repulsive force acts between the particles in contact in addition, given by

$f_{\mathrm{n}}=k \delta+\gamma_{\mathrm{o}} \dot{\delta}$,

where $k$ is the elastic stiffness, $\gamma_{0}$ is the viscous damping coefficient, and $\delta$ is the overlap between the particles. The normal contact forces for the liquid bridge model are explained in Sect. 2.3.1

\subsubsection{Liquid bridge capillary force model}

The capillary pressure difference sustained across the liquidair interface due to surface tension can be described by the non-linear Laplace-Young equation [22]. This relates the pressure difference to the shape of the surface under the criterion of minimum Gibbs free energy [35]. The capillary force in a pendular bridge originates from the axial component of this force. Another component that contributes to the capillary force is due to the hydrostatic pressure. Many previous studies have calculated capillary forces based on the numerical solution of the Laplace-Young equation and also reported experimental results $[19,22]$. The magnitude of liquid bridge capillary force depends on the volume of the liquid bridge between the particles, the contact angle $\theta$, surface tension $\gamma$, the effective radius of the particles $r$, and the separation distance $S, S=-\delta$. With these parameters, we approximate the inter-particle force $f_{\mathrm{c}}$ of the capillary bridge according to [19]. The experimental results are fitted by a polynomial to obtain the dependence of capillary forces on the scaled separation distance. During approach of the particles as indicated by the loading branch in Fig. 2, the normal contact force for this model is given by

$f= \begin{cases}0 & \text { if } \delta<0 ; \\ -f_{\mathrm{a}}{ }^{\max }+f_{\mathrm{n}} & \text { if } \delta \geq 0 .\end{cases}$

During separation of the particles as indicated by the unloading branches in Fig. 2, the normal contact force for this model is given by 
$f= \begin{cases}0 & \text { if } \delta<-S_{\mathrm{c}} ; \\ -f_{\mathrm{a}} & \text { if }-S_{\mathrm{c}} \leq \delta<0 \\ -f_{\mathrm{a}}{ }^{\max }+f_{\mathrm{n}} & \text { if } \delta \geq 0,\end{cases}$

where $f_{\mathrm{n}}$ is the normal repulsive force given by Eq. (9). The adhesive force for the liquid bridge model is the capillary force given by

$f_{\mathrm{a}}=\left(f_{\mathrm{a}}\right)_{\text {liq }}=\frac{\left(f_{\mathrm{a}}{ }^{\max }\right)_{\text {liq }}\left(\frac{2 r}{d_{\mathrm{p}}}\right)}{1+1.05 \bar{S}+2.5 \bar{S}^{2}}$,

where the separation distance is normalized as $\bar{S}=$ $S \sqrt{\left(r / V_{\mathrm{b}}\right)}, S$ being the separation distance. The maximum capillary force between the particles when they are in contact $(S=0)$ is given by

$\left(f_{\mathrm{a}}{ }^{\max }\right)_{\text {liq }}=\pi d_{\mathrm{p}} \gamma \cos \theta$,

where $d_{\mathrm{p}}$ is the mean particle diameter. The effective radius of two interacting spherical particles of different sizes can be estimated as the harmonic mean of the two particle radii according to the Derjaguin approximation [36], yielding the effective radius:

$r=\frac{2 r_{i} r_{j}}{r_{i}+r_{j}}$

however, the mean size is not varied here. This model equation is applicable for mono-disperse particles [12,19], which has been actually extended to poly-disperse system of particles Ref. [14]. As proposed by [37], the critical separation distance $S_{\mathrm{c}}$ between the particles before the bridge ruptures is given by

$\left(S_{\mathrm{c}}\right)_{\text {liq }}=\left(1+\frac{\theta}{2}\right) V_{\mathrm{b}}^{1 / 3}$

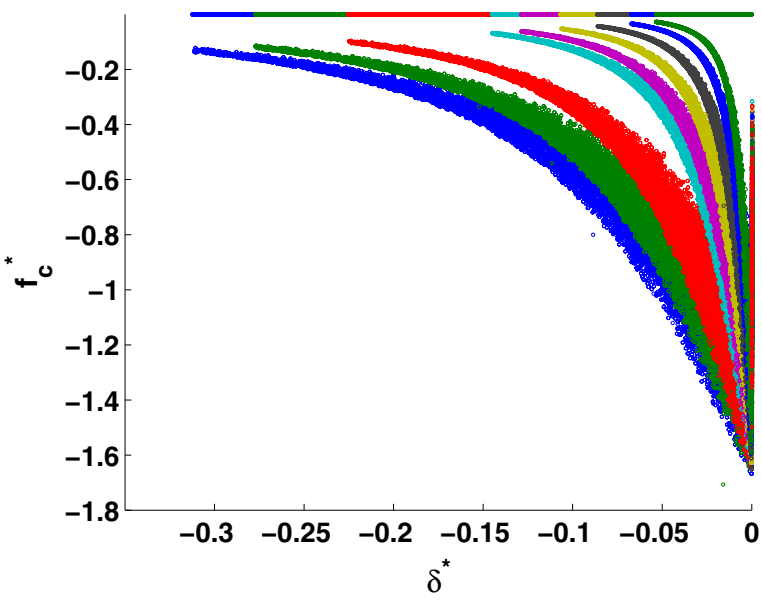

Fig. $3 f_{\mathrm{c}}{ }^{*}$ as a function of $\delta^{*}$. Different colors represent different liquid bridge volumes. (Color figure online)
The liquid bridge capillary force as a function of separation distance is shown in Fig. 3 for different liquid bridge volumes. The capillary force decreases in magnitude with increasing separation distance between the particles till the bridge ruptures. This is in agreement with the experimental measurements of capillary force done by a microbalance [19]. Experimental measurements of capillary force by [38] for smaller particle size using an atomic force microscope (AFM) show that the attraction force first increases and then decreases with separation distance till rupture. The high resolution for measurements provided by AFM at the nanometer scale is able to capture this. The increase in force with increase in separation distance is due to the influence of contact line pinning in wetting hysteresis which is prevalent on heterogeneous surface [39]. We assume the surface of particles to be homogeneous without defects, thereby neglecting the effect of pinning and only slipping occurs. [13] compares the macroscopic results for different liquid capillary bridge models and shows that they are in agreement, except the model of [32]. The rupture distance is proportional to $V_{\mathrm{b}}^{1 / 3}$ as stated in Eq. (15).

\subsubsection{Linear irreversible contact model}

In Sect. 4, we introduce a simple linear irreversible contact model as proposed by [23] and shown in Fig. 4 which can be compared with the non-linear liquid bridge interaction model. For the linear irreversible contact model, the normal forces between particles during approach and separation are given by Eqs. (10) and (11), respectively, where for the linear irreversible contact model,

$$
\begin{aligned}
f_{\mathrm{a}} & =\left(f_{\mathrm{a}}\right)_{\operatorname{lin}}=\left(f_{\mathrm{a}}^{\max }\right)_{\operatorname{lin}}+k_{\mathrm{c}} \delta, \\
\left(S_{\mathrm{c}}\right)_{\operatorname{lin}} & =\left(f_{\mathrm{a}}^{\mathrm{max}}\right)_{\operatorname{lin}} / k_{\mathrm{c}},
\end{aligned}
$$

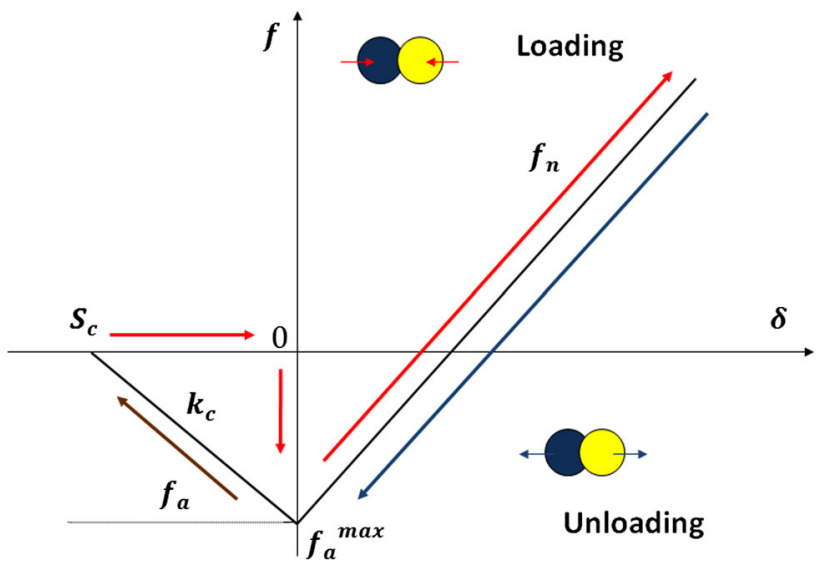

Fig. 4 Linear irreversible contact model. The red lines represent the loading direction, the blue line represents the unloading direction when the particles are in contact, and the brown line represents the unloading for the non-contact particles with short-range interaction force. (Color figure online) 
Table 2 Non-dimensionalization of parameters

\begin{tabular}{llll}
\hline Parameter & Symbol & Scaled term & Scaling term \\
\hline Capillary force & $f_{\mathrm{c}}$ & $f_{\mathrm{c}}{ }^{*}$ & $f_{\mathrm{g}}$ \\
Particle overlap & $\delta$ & $\delta^{*}$ & $d_{p}$ \\
Shear stress & $\tau$ & $\tau^{*}$ & $f_{\mathrm{g}} / d_{\mathrm{p}}{ }^{2}$ \\
Pressure & $P$ & $P^{*}$ & $f_{\mathrm{g}} / d_{\mathrm{p}}{ }^{2}$ \\
Steady-state cohesion & $c$ & $c^{*}$ & $f_{\mathrm{g}} / d_{\mathrm{p}}{ }^{2}$ \\
Liquid bridge volume & $V_{\mathrm{b}}$ & $V_{\mathrm{b}}{ }^{*}$ & $d_{\mathrm{p}}{ }^{3}$ \\
Surface tension & $\gamma$ & $\gamma^{*}$ & $f_{\mathrm{g}} / d_{\mathrm{p}}$ \\
Rupture distance & $S_{\mathrm{c}}$ & $S_{\mathrm{c}}{ }^{*}$ & $d_{\mathrm{p}}$ \\
Torque & $T_{\mathrm{z}}$ & $T_{\mathrm{z}}{ }^{*}$ & $f_{\mathrm{g}} d_{\mathrm{p}}$ \\
Angular rotation & $\theta_{\mathrm{rot}}$ & $\theta_{\mathrm{rot}}{ }^{*}$ & $2 \pi$ \\
Adhesive energy & $E$ & $E^{*}$ & $f_{\mathrm{g}} d_{\mathrm{p}}$ \\
\hline
\end{tabular}

where $\left(f_{\mathrm{a}}^{\max }\right)_{\text {lin }}$ is the maximum adhesive force and $k_{\mathrm{c}}$ is the adhesive stiffness. The tangential force contact model is explained in details in our earlier studies [27].

\subsection{Dimensional analysis}

To formulate all the modeling equations in a constructive way, we express them in non-dimensionalized form. All the length scale parameters are scaled by the mean particle diameter $d_{\mathrm{p}}=2.20 \mathrm{~mm}$. The forces are scaled in terms of the gravitational force acting on a single particle $f_{\mathrm{g}}=V_{\mathrm{p}} \rho g \approx$ $1.0939 \times 10^{-4} \mathrm{~N}$. Table 2 shows all the parameters in their dimensionless form and the corresponding scaling terms used in the equations. The angular rotation of the shear cell after a given time to study the dynamic evolution of torque is scaled in terms of radians covered in one complete rotation $(2 \pi)$. The dynamics of the system can be characterized by the time scale defined by the contact duration between two particles $t_{\mathrm{c}}=\sqrt{m_{\mathrm{p}} / k}$, where $m_{\mathrm{p}}$ is the mean mass of a particle. Since we do all our macro-rheology analysis in steady state, characterization of dynamics of the system is not required. The main objectives of non-dimensionalization are to simplify the equations in terms of unit less quantities and define the system intrinsically.

\section{Micro-macro transition}

To extract the macroscopic properties, we use the spatial coarse-graining approach detailed in [40-42]. The averaging is performed over toroidal volume, over many snapshots of time assuming rotational invariance in the tangential $\phi$ direction. The averaging procedure for a three-dimensional system is explained in $[41,42]$. This spatial coarse-graining method was used earlier in $[1,23,27,28,42]$. The simulation is run for $200 \mathrm{~s}$ and temporal averaging is done when the flow is in steady state, between 80 and 200 s, thereby disregarding the transient behavior at the onset of the shear.

\subsection{Steady-state cohesion and its correlation with liquid bridge volume and surface tension}

In earlier studies [12,27,40,41], the shear band region was identified by the criterion of large strain rate, e.g., higher than a critical strain rate of $0.08 \mathrm{~s}^{-1}$. In this paper, the shear band center region is defined by strain rates higher $80 \%$ of the maximum for different heights in the shear cell. Figure 5 displays the dependence of scaled yield stress $\tau^{*}$ for the particles in the shear band region on scaled pressure $P^{*}$ for $75 \mathrm{nl}$ liquid bridge volume. A linear trend is observed neglecting the different behaviors for data at very low pressure $\left(P^{*}<4.42\right)$. This is fitted well by a linear function:

$\tau^{*}=\mu P^{*}+c^{*}$,

where $\mu$ is the macroscopic friction coefficient and $c^{*}$ is the steady-state cohesion obtained from the plot. Next, we fit the data for shear stress as a function of pressure as given by Eq. (18) and obtain the value of steady-state cohesion and macroscopic friction $\mu$. The macroscopic friction coefficient is constant for lower surface tension, including $\gamma^{*}=0$ for linear elastic model (not shown in figure), but increases for $\gamma^{*} \gtrsim 2$ for a given liquid bridge volume as shown in Fig. 6. When the surface tension of the material is very high $\left(\gamma^{*} \gtrsim 20\right)$, materials protrude out of the top surface to form a hump in the region of the shear band (data not shown). For our analysis of surface tension in the range $0.020-0.060 \mathrm{~N} \mathrm{~m}^{-1}$, the macroscopic friction coefficient is constant at $\mu \simeq 0.15$.

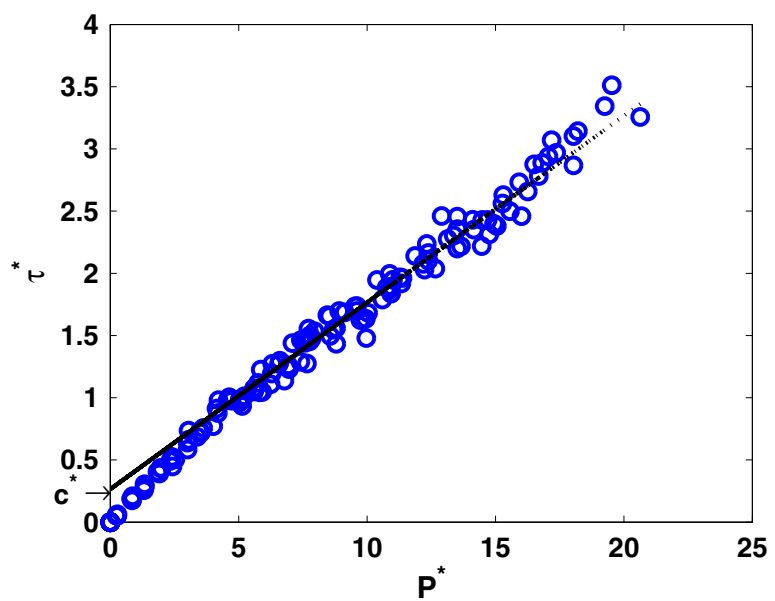

Fig. 5 Shear stress $\tau^{*}$ plotted against pressure $P^{*}$. The dotted line represents the fitting function as given by Eq. (18) for $P^{*}>4.42 \mathrm{~Pa}$ where $\mu=0.15$ is the macroscopic friction coefficient, $c^{*}=0.2655$ for $V_{\mathrm{b}}=75 \mathrm{nl}$ and $\gamma=0.020 \mathrm{Nm}^{-1}$ 


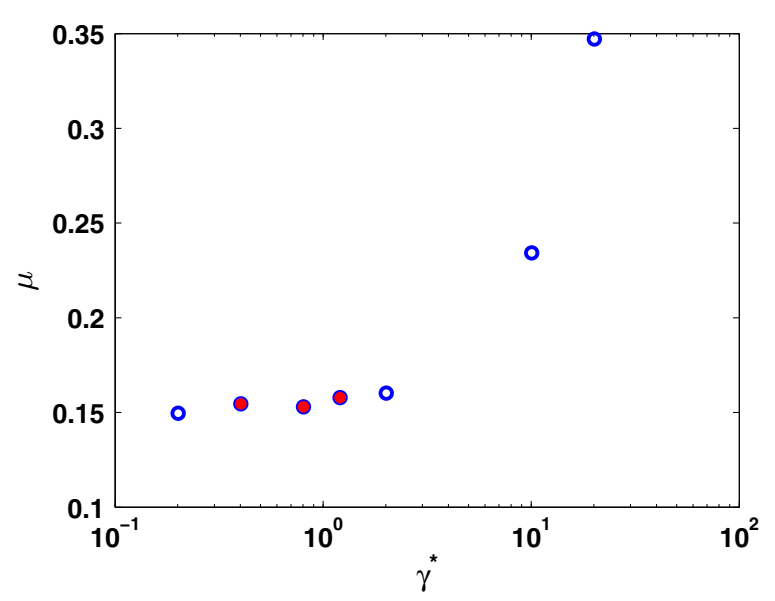

Fig. 6 Macroscopic friction coefficient $\mu$ as a function of $\gamma^{*}$ for $V_{\mathrm{b}}=$ $75 \mathrm{nl}$. The solid symbols represent the range of surface tension for our simulations below

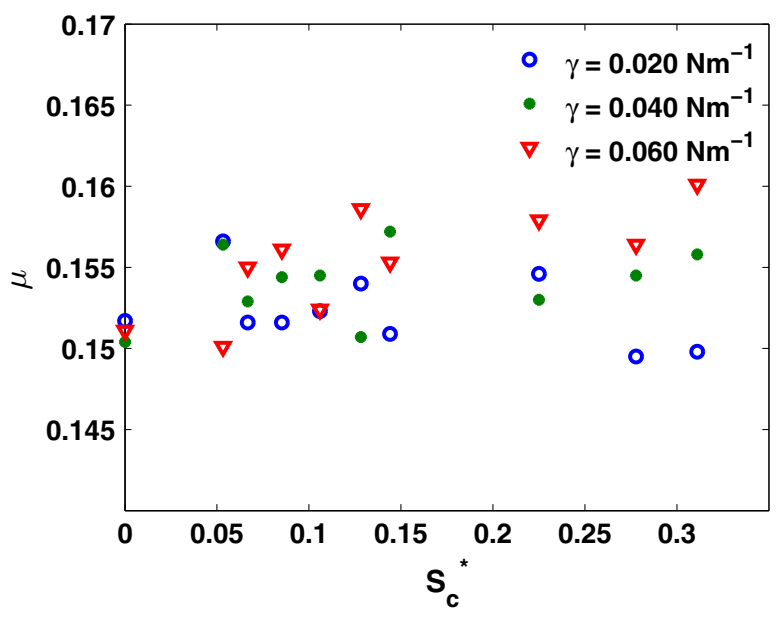

Fig. 7 Macroscopic friction coefficient $\mu$ as a function of $S_{\mathrm{c}}{ }^{*}$ for $\gamma=$ $0.020 \mathrm{Nm}^{-1}$

In this range, the macroscopic friction coefficient is also independent of the liquid bridge volume as shown in Fig. 7.

For dry cohesionless systems, the dependence of shear stress on pressure is linear without an offset, i.e., $c^{*}=0$. In the presence of interstitial liquid between the particles in the pendular regime, cohesive forces increase with increasing liquid bridge volume. This results in a positive steady-state cohesion $c^{*}$ as given by Eq. (18), see Fig. 5.

Earlier studies on wet granular materials have shown that the presence of liquid bridges between the particles results in an increasing steady-state cohesion of the materials $[12,13,15,27]$. Our earlier studies show that the steady-state cohesion $c^{*}$ increases non-linearly with increasing liquid bridge volume. Here, the steady-state cohesion is studied in more detail, including very small liquid bridge volumes, including the (practically impossible) limit of $0 \mathrm{nl}$ liquid bridge volume as given in Eq. (3). Note that there is a finite cohesive strength for $V_{\mathrm{b}} \rightarrow 0 \mathrm{nl}$ liquid bridge volume. This
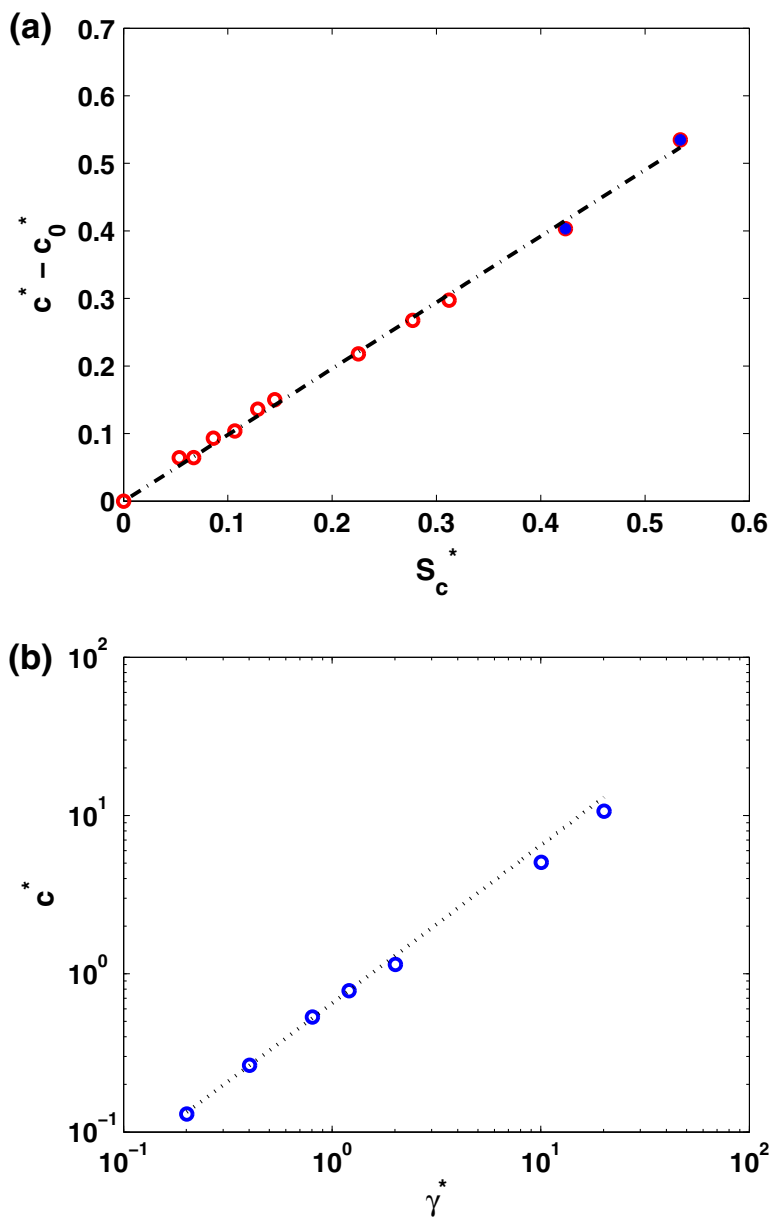

Fig. 8 a $c^{*}-c_{0}{ }^{*}$ as a function of $S_{c}{ }^{*}$ for $\gamma=0.020 \mathrm{Nm}^{-1}$. The dotted line represents the fitting function given by Eq. (20). The data with solid symbols represent the liquid bridge volume outside the pendular regime. b $c^{*}$ as a function of $\gamma^{*}$ for $V_{\mathrm{b}}=75 \mathrm{nl}$. The dotted line represents the fitting function given by Eq. (21)

is due to the microscopic capillary bridge force that acts between particles even at $0 \mathrm{nl}$ liquid bridge volume as given by Eq. (13). This is called the steady- state critical cohesion $c_{0}{ }^{*}$ for a given surface tension of liquid. This value depends on the maximum force acting between two particles when they are in contact as given by Eq. (13). The additional cohesion for higher liquid bridge volume is due to the non-contact capillary forces between the particles that are active up to the distance when the liquid bridge ruptures. This is dependent on the surface tension of the liquid and the volume of the liquid bridge. Thus, the steady-state cohesion of granular materials for a given liquid bridge volume can be written as follows:

$c^{*}=c_{0}^{*}+c^{\prime *}$,

where $c^{\prime *}$ is the additional cohesion for liquid bridge volume $V_{b}>0$. Figure 8a shows $\left(c^{*}-c_{0}{ }^{*}\right)$ as a linear function of $S_{c}{ }^{*}$, fitted by

$c^{*}-c_{0}{ }^{*}=a S_{c}^{*}$, 
where $a=0.9805$ for $\gamma=0.020 \mathrm{Nm}^{-1}$. In the next section, we study the dependence of this constant on the surface tension of liquid.

Figure $8 \mathrm{~b}$ shows the dependence of steady-state cohesion on $\gamma^{*}$ for $V_{\mathrm{b}}=75 \mathrm{nl}$. The steady-state cohesion can be described by

$\ln c^{*}=\alpha \ln \gamma^{*}+k$,

where $\alpha \approx 1.00, k=-0.4240$. Therefore, the steady- state cohesion is linearly proportional to the surface tension and can be written as follows:

$c^{*}=b \gamma^{*}$,

where $b=\exp (k)$. The above equation is valid in the limit of zero surface tension $\left(\gamma^{*}=0\right)$ which represents the simple linear elastic contact model. For higher surface tension of liquid, the results deviate from the fitted function of linear dependence as seen from Fig. 8b. As given by Eqs. (20) and (21), the steady-state cohesion is dependent on liquid bridge volume expressed in terms of maximum interaction distance $S_{c}{ }^{*}$ between the particles and the maximum adhesive force expressed in terms of surface tension of the liquid $\gamma^{*}$. So, in the later sections of this paper, we study the dependence of macroscopic parameters on the microparameters $S_{c}{ }^{*}$ representing scaled rupture distance and $\gamma^{*}$ representing scaled maximum force for all contact models.

Figure 9 shows the dependence of $c^{*}-c_{0}{ }^{*} / \gamma^{*}$ on $S_{c}{ }^{*}$ for a different surface tensions of liquid. The scaled steadystate cohesion is a linearly dependent on the rupture distance as shown in the figure. This can be fitted by a straight line equation given by

$\frac{c^{*}-c_{0}^{*}}{\gamma^{*}}=\frac{c^{*}-c_{0}^{*}}{\left(f_{\mathrm{a}}^{\max }\right)_{\mathrm{liq}}{ }^{*} /(\pi \cos \theta)}=p S_{c}^{*}$,

where $p=2.1977$ as obtained from the fitting shown in Fig. 9; the offset is very small and can be neglected.

This subsection shows that the macroscopic characteristics of the liquid bridge model are determined by the maximum interacting force between the particles and the rupture distance. The steady-state cohesion scales linearly with the surface tension of liquid, i.e., the maximum force between the particles. For a given maximum force, the cohesion scaled with the surface tension of liquid is also a linear function of the rupture distance of the liquid bridge.

\subsection{Macroscopic torque analysis from the microscopic parameters}

The strength, cohesion, and flow properties of granular materials are strongly influenced by the presence of capillary



Fig. $9 \frac{c^{*}-c_{0}{ }^{*}}{\gamma^{*}}$ as a function of $S_{c}^{*}$ for a different surface tension of liquid. The dotted line represents the fitting function given by Eq. (23)

cohesion. Due to the cohesive properties of these wet materials, the shear stress increases and, as a result, partially saturated wet materials require higher torques for deformation (shear), e.g., in a shear cell. Loosely speaking, torque is a measure of the shear stress or force acting on the particles at the wall and thus can be used to find an estimate of shear stress in the shear band. To study solely the effect of capillary cohesion on the torque, the other parameters like the particle friction are kept very small in our simulations, with $\mu_{\mathrm{g}}=0.01$. Earlier studies $[13,27,43,44]$ show that the average torque acting on the rotating part of the shear cell increases with increasing moisture content. In this section, we perform a detailed analysis of the macroscopic torque as a function of the microparameters in order to understand its connection with the steady-state cohesion of the material.

The walls and the bottom plates of the shear cell consist of particles with a prescribed position. The particles forming the inner wall are stationary, while the particles forming the outer wall rotate around the $z$-axis with frequency $f_{\text {rot }}$. All the particles forming the inner and outer wall are identified as $\mathcal{C}_{\text {inner }}$ and $\mathcal{C}_{\text {outer }}$, respectively. The macroscopic torque is calculated based on the contact forces on the fixed particles on the moving (outer) and stationary (inner) parts of the shear cell. Thus, the net inner and outer torque are calculated by summing up the torques for all the contacts with respect to the axis of rotation of the shear cell. The net torque is obtained from the difference between the outer wall torque and the inner wall torque. We multiply the total torque by a factor of $2 \pi /(\pi / 6)$ in order to get the torque for the whole system from the obtained torque of our simulations in a $30^{\circ}$ section. Thus, the torque is given by 


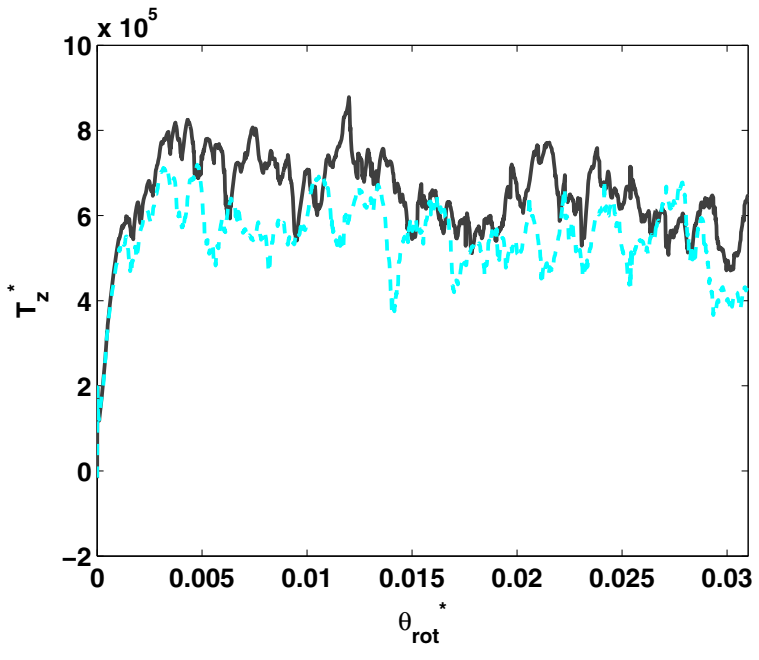

Fig. $10 T_{z}{ }^{*}$ as a function of scaled angular rotation $\theta_{\text {rot }}{ }^{*}$ for surface tension of liquid $\gamma=0.020 \mathrm{Nm}^{-1}$ for $V_{\mathrm{b}}=4.2 \mathrm{nl}$ (dash cyan) and $V_{\mathrm{b}}=200 \mathrm{nl}($ solid black $)$

$$
\begin{aligned}
\mathbf{T}=\frac{2 \pi}{\pi / 6}[ & \left(\sum_{i=1}^{N} \sum_{j \in \mathcal{C}_{\text {outer }}} \mathbf{c}_{i, j} \times \mathbf{f}_{i, j}\right)- \\
& \left.\left(\sum_{i=1}^{N} \sum_{j \in \mathcal{C}_{\text {inner }}} \mathbf{c}_{i, j} \times \mathbf{f}_{i, j}\right)\right],
\end{aligned}
$$

where $N$ represents the number of particles, $\mathbf{c}_{i j}$ is the position of the contact point, and $\mathbf{f}_{i j}$ is the interaction force. Only the $z$-component of the torque vector $\left(T_{z}\right)$ is of interest as required for shearing the cell in angular direction.

We compare our results with the experimental results as given by [45] from the evolution of torque as a function of the angular rotation as shown in Fig. 10. This is in good agreement with the magnitude and angular rotation required for steady-state torque evolution as given in [45], considering the different rotation rates and different frictions in the systems.

Figure 11 shows $T_{z}^{*}$ as a function of $\gamma^{*}$ for different liquid bridge volumes. We observe that the resultant torque depends linearly in the surface tension of the liquid. The fit parameter $l$ from the figure, the rate of increase of torque with surface tension, depends on the liquid bridge volume.

Next, we compare the results of the steady-state cohesion as obtained from the fitting function explained in Sect. 3.1 with the calculated (measured) torque. We write the scalar form of the torque on the wall derived from steady- state cohesion as $T_{\mathrm{Z}}^{\text {macro }}$

$T_{\mathrm{Z}}^{\text {macro }}=\left[\int_{A_{o}} r \mathrm{~d} A-\int_{A_{i}} r \mathrm{~d} A\right]\left(\mu P_{\mathrm{avg}}+c\right)$,

where $A_{\mathrm{o}}$ denotes the outer wall surface, $A_{\mathrm{i}}$ denotes the inner wall surface, and $P_{\text {avg }}$ is the mean pressure inside the shear band approximately $250 \mathrm{~Pa}$ for a filling height of $39 \mathrm{~mm}$. Equation (25) can be simplified to the form:

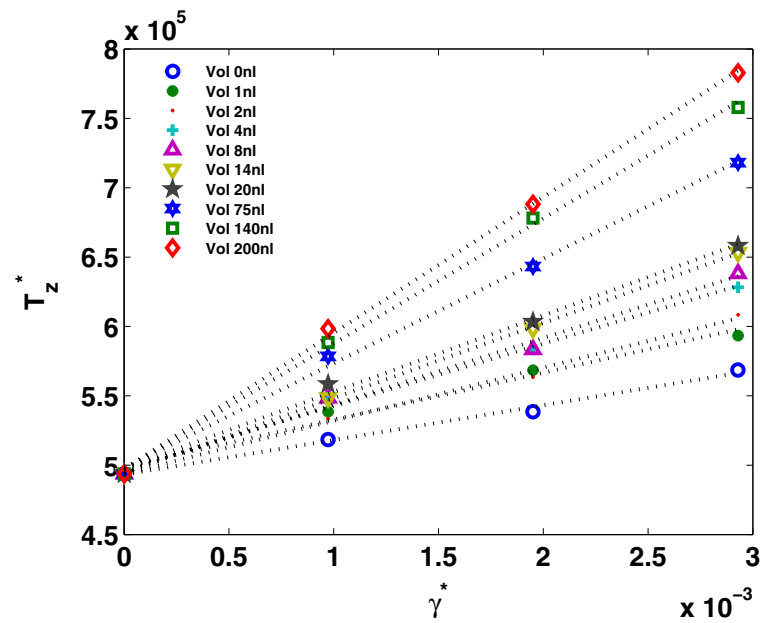

Fig. $11 T_{z}^{*}$ as a function of $\gamma^{*}$. The dotted lines represent the fitting functions for different liquid bridge volumes given by equation $T_{z}{ }^{*}=$ $l \gamma^{*}+t$ where $t=4.964 \times 10^{5}$ and $l$ increases with increasing liquid bridge volume

$T_{\mathrm{z}}^{\text {macro }}=M\left(\mu P_{\mathrm{avg}}+c\right)$,

where $M=\left[2 \pi H\left(R_{\mathrm{o}}{ }^{2}-R_{\mathrm{i}}{ }^{2}\right)+\frac{2}{3} \pi\left(R_{\mathrm{o}}{ }^{3}+R_{\mathrm{i}}{ }^{3}-2 R_{\mathrm{S}}{ }^{3}\right)\right]$ $\approx 0.0031 \mathrm{~m}^{3}$ for the given geometry is equal to fitting parameter $t /\left(\mu P_{\text {avg }}\right), t$ is the fit parameter, see Fig. 11. Assuming $T_{z}=T_{z}{ }^{\text {macro }}$, an equivalent steady-state cohesion as obtained from the calculated torque can be given as follows:

$c_{\mathrm{eq}}=T_{z} / M-\mu P_{\mathrm{avg}}$

Figure 12 shows the dependence of the non-dimensionalized value $c_{\mathrm{eq}}{ }^{*}-\left(c_{\mathrm{eq}}\right)_{0}{ }^{*} / \gamma^{*}$ on $S_{c}{ }^{*}$ for a different surface tension. $\left(c_{\mathrm{eq}}\right)_{0}^{*}$ is the equivalent steady-state cohesion as obtained from Eq. (27) for the torque of a $0 \mathrm{nl}$ liquid bridge. This can be fitted by a straight line:

$\frac{c_{\mathrm{eq}}{ }^{*}-\left(c_{\mathrm{eq}}\right)_{0}{ }^{*}}{\gamma^{*}}=e S_{c}{ }^{*}$,

where $e=2.0062$ is a fit parameter, see Fig. 12, and the offset is very small and can be neglected. Equation (28) shows equivalent steady- state cohesion as obtained from the torque is also linearly dependent on $S_{c}{ }^{*}$. The fitting parameter $e$ of this equation shows a close similarity with the fitting parameter $p$ of Eq. (23). Alternatively, Fig. 13 shows a comparison of the two torques given by the scalar $z$-component of Eqs. (24) and (26) for surface tension of liquid $0.020 \mathrm{~N} \mathrm{~m}^{-1}$. These results show that the steady-state cohesion and torque are related by Eq. (26).

In conclusion, this subsection shows that the measured torque can be translated to the local steady-state macrorheology parameters via a simple factor $M$ (a measure of 


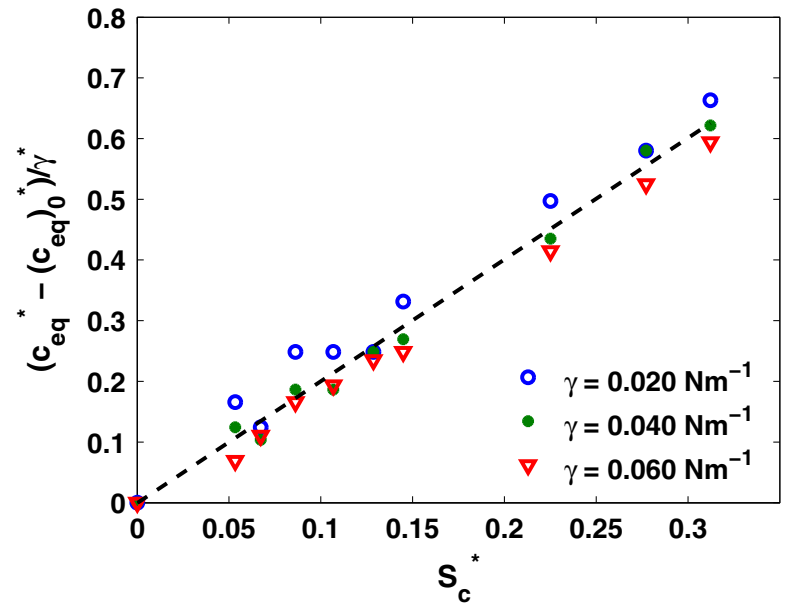

Fig. $12 \frac{c_{\mathrm{eq}}{ }^{*}-\left(c_{\mathrm{eq}}\right)_{0}{ }^{*}}{\gamma^{*}}$ as a function of $S_{c}{ }^{*}$ for different surface tensions of liquid where $c_{\mathrm{eq}}$ is given by Eq. (27). The dotted line represents the fitting function as given by Eq. (28)

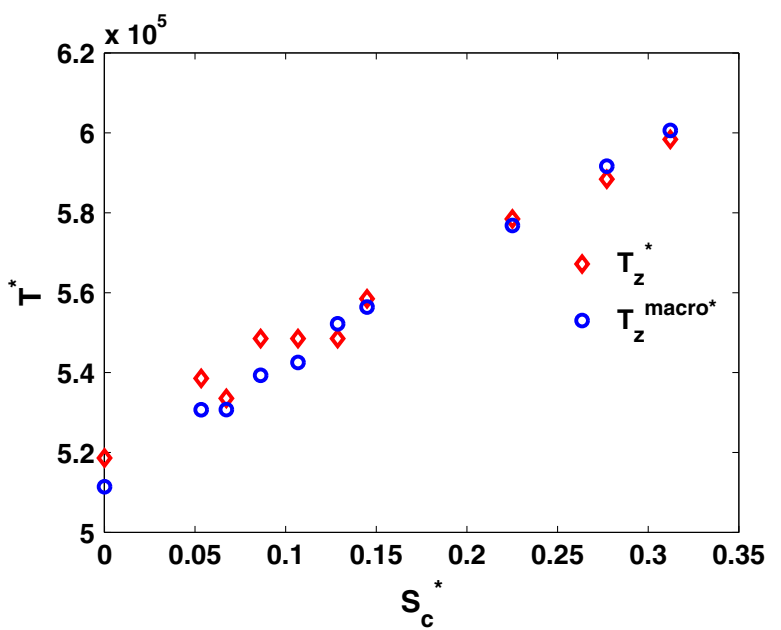

Fig. 13 Torque calculated numerically scaled as $T_{z}{ }^{*}$ as compared with the scalar form of scaled macro-torque $T_{z}^{\text {macro* }}$ as calculated from the wall shear stress as given by Eq. (26)

the resultant arm-length times surface area) which depends only on the geometry of the system.

\section{An analogous linear irreversible contact model for cohesive particles}

In this section, we aim to determine the key microscopic parameters for a linear irreversible contact model [23] that is macroscopically analogous to the liquid bridge contact model used before. An explanation of the linear irreversible contact model is given in [23]. Unlike the liquid bridge contact model, the force for the linear irreversible contact model is simple and faster to compute. Figure 14 shows the force-overlap distribution for the two contact models show-

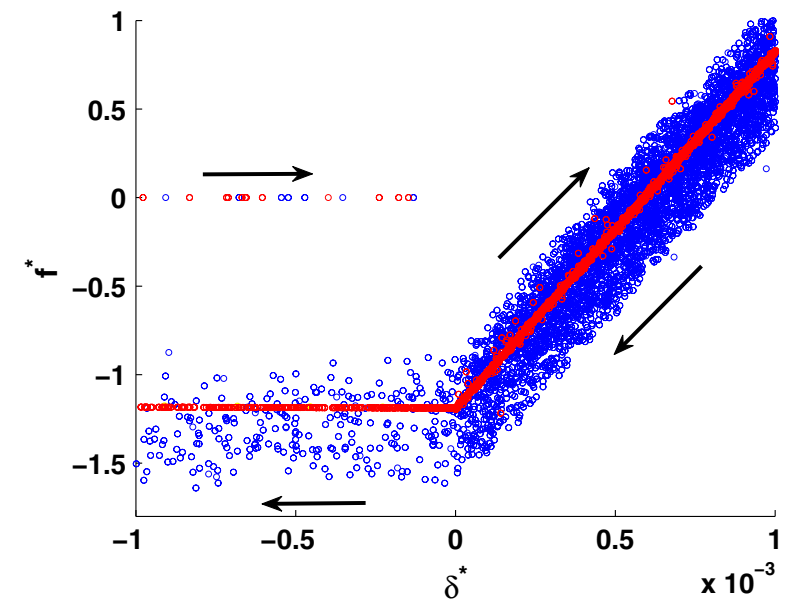

Fig. 14 Force-overlap diagram for the liquid bridge model (blue) as compared with the linear irreversible contact model (red). The arrow shows the loading and the unloading directions for all forces. The schematic diagram for the same is given in Figs. 2 and 4, respectively. (Color figure online)

ing the loading and unloading directions of forces which are reversible at $\delta^{*}>0$ and irreversible at $\delta^{*}<0$.

As discussed in Sect. 3.1, the steady-state cohesion for the liquid bridge model is controlled by the rupture distance of the liquid bridge, which is proportional to the liquid bridge volume, and the magnitude of the maximum interaction force, which is governed by the surface tension of the liquid. Assuming that the non-linear liquid bridge capillary force can be replaced by a simple irreversible linear adhesive force between the particles with the same macrocharacteristics, we compare the steady-state cohesion of the two models in Sect. 4.1.

\subsection{Equal maximum force and interaction distance}

The key parameters that define the cohesive force of a linear irreversible contact model are the maximum adhesive force and the adhesive stiffness, see Eq. (16). Several simulations have been run for the linear irreversible contact model in the same numerical set-up with the same maximum adhesive force as used in the liquid bridge model $\left(\left(f_{\mathrm{a}}{ }^{\max }\right)_{\text {liq }}=\left(f_{\mathrm{a}}{ }^{\max }\right)_{\text {lin }}\right)$ and adhesive stiffness that would result in the same interaction range for different liquid bridge volumes for a different surface tension of liquid. The forceoverlap for contacts with $\delta^{*}<0$ for the two comparable contact models with equal interaction distance is shown in Fig. 15. The adhesive stiffnesses that are equivalent to the liquid bridge volumes as given by Eq. (3) for surface tension $\gamma=0.020 \mathrm{~N} \mathrm{~m}^{-1}$ for equal interaction distance are given by

$$
\begin{aligned}
& k_{\mathrm{a}} \in\{0.21,0.26,0.41,0.46 \text {, } \\
& 0.56,0.69,0.88,1.11, \infty\} \mathrm{N} \mathrm{m}^{-1}
\end{aligned}
$$




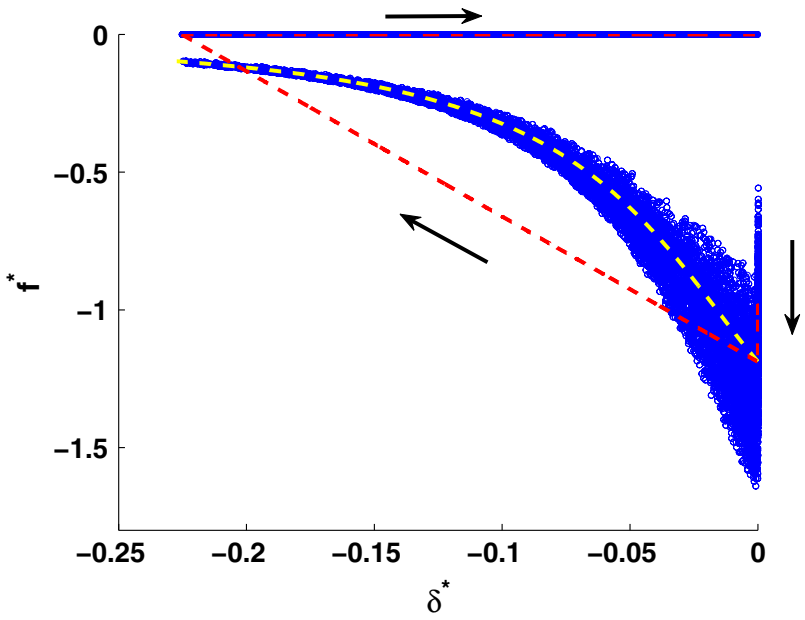

Fig. 15 Scaled adhesive force $f^{*}\left(f_{\mathrm{a}}{ }^{*}\right.$ for linear adhesive model and $f_{\mathrm{c}}{ }^{*}$ for liquid bridge model) as a function of $\delta^{*}$ for the linear irreversible contact model (red), compared with the liquid bridge model (blue), for equal maximum force and equal interaction distance. The yellow line represents the force for the liquid bridge contact model for mean particle diameter $d_{p}$ as a function of $\delta^{*}$. The arrow shows the loading and the unloading directions for the short-range forces. (Color figure online)

The results for the steady-state cohesion $c^{*}$, as scaled by $\gamma^{*}$ for the liquid bridge model and the linear irreversible model are shown in Fig. 17. The results are not really analogous as seen from the figure as the intercepts for the fitting lines of the two models are different, while they are parallel. The fitting parameters for the relation

$\frac{c^{*}-c_{0}^{*}}{\gamma^{*}}=g S_{c}^{*}+h$

are $g=2.1716$ and $h \approx 0$ for the liquid bridge contact model, $g=2.0984$ and $h=0.2226$ for the linear irreversible contact model.

So for a given liquid bridge volume and a given surface tension of liquid, the linear irreversible contact model with the same maximum force and same interaction distance has a higher cohesion.

\subsection{Equal maximum force and adhesive energy}

Equal maximum force and interaction distance was discussed in Sect. 4.1, but here the steady-state cohesion for the two models with an equal maximum adhesive force and equal adhesive energy $E^{*}$ is considered. The adhesive energy for a given contact model is obtained by the total area under the force-overlap distribution, see Fig. 16. A linear model analogous to the liquid bridge contact model is obtained with the equal maximum force with surface tension $\gamma=0.020 \mathrm{Nm}^{-1}$ and the adhesive stiffness adjusted to have the equal adhesive energy:

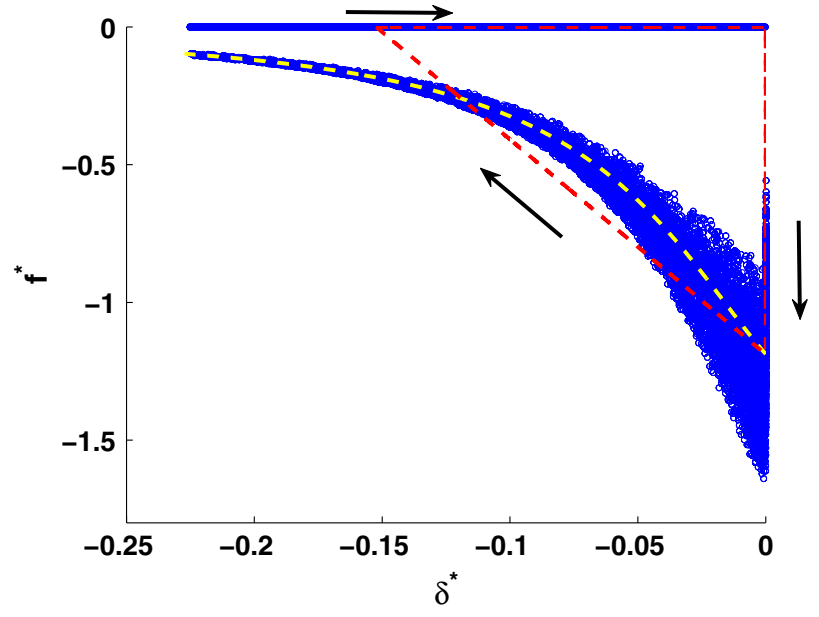

Fig. 16 Scaled adhesive force $f^{*}\left(f_{\mathrm{a}}{ }^{*}\right.$ for linear adhesive model and $f_{\mathrm{c}}{ }^{*}$ for liquid bridge model) as a function of $\delta^{*}$ for linear the irreversible contact model (red), compared with the liquid bridge model (blue), for equal maximum force and equal adhesive energy dissipated per contact. The yellow line represents the force for the liquid bridge contact model for mean particle diameter $d_{\mathrm{p}}$ as a function of $\delta^{*}$. The arrow shows the loading and the unloading directions for the short-range forces. (Color figure online)

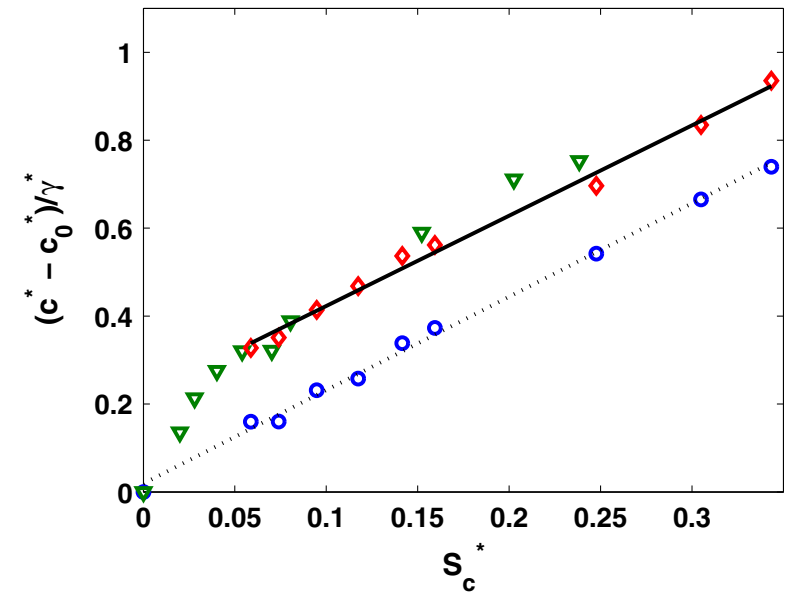

Fig. $17 \frac{c^{*}-c_{0}^{*}}{\gamma^{*}}$ as function of $S_{c}{ }^{*}$ for the liquid bridge model (blue) and the linear irreversible model with equal interaction distance (red) and equal adhesive energy dissipated per contact (green) for $\gamma=$ $0.020 \mathrm{~N} \mathrm{~m}^{-1}$. The dotted and the solid lines represent the fitting function given by Eq. (30). (Color figure online)

$$
\begin{aligned}
& k_{a} \in\{0.25,0.29,0.39,0.74,0.84 \\
&1.10,1.49,2.11,2.95, \infty\} \mathrm{Nm}^{-1}
\end{aligned}
$$

The force-overlap for contacts with $\delta^{*}<0$ for the two comparable contact models with equal adhesive energy is shown in Fig. 16. Figure 17 shows the dependence of $c^{*}-c_{0}{ }^{*} / \gamma^{*}$ on rupture distance $S_{c}{ }^{*}$ for the liquid bridge model (blue), compared with the two cases of the linear irreversible contact model with equal interaction distance (red) and equal adhesive energy dissipated per contact (green). The linear irreversible model with equal energy has a lower inter- 


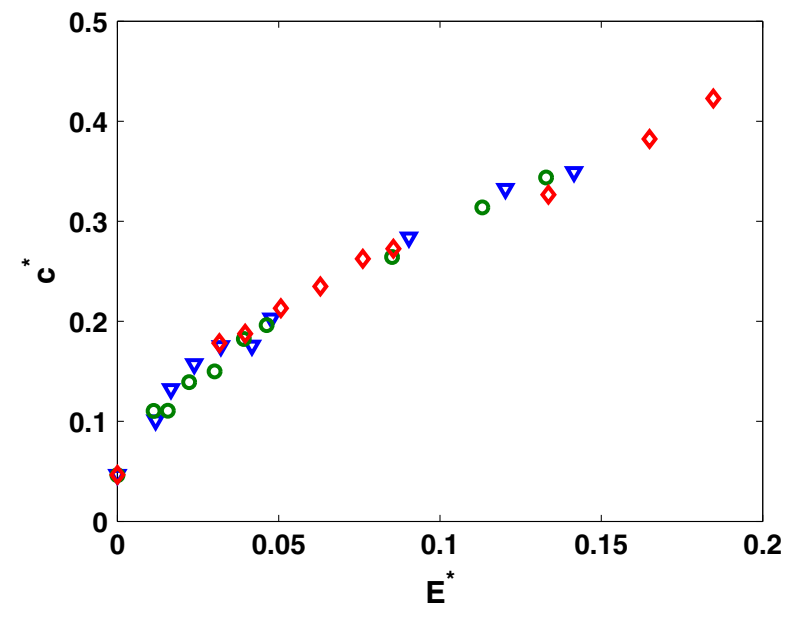

Fig. $18 c^{*}$ as a function of $E^{*}$ for the liquid bridge model (blue) and the linear irreversible model with equal interaction distance $($ red $)$ and equal adhesive energy dissipated per contact (green) for $\gamma=0.020 \mathrm{Nm}^{-1}$. (Color figure online)

action distance. The functional behavior of the steady-state cohesion using the linear irreversible contact model for small interaction range can be understood from this. As observed from Fig. 17, the cohesion is a non-linearly dependent on the rupture distance $S_{c}{ }^{*}$ at low interaction distance and becomes linear for higher range.

Figure 18 shows the dependence of steady-state cohesion on total adhesive energy for the liquid bridge model, compared with the two cases of linear irreversible contact model with equal interaction distance (red) and equal adhesive energy dissipated per contact (green). As seen from the figure, for a given maximum force which is determined by the surface tension of the liquid, the steady-state cohesion $c^{*}$ is equal for the liquid bridge model and the linear irreversible model with equal energy. The steady-state cohesion for the linear irreversible model with equal interaction distance is higher as it has higher adhesive energy than the liquid bridge model. However, all the data for the three cases as explained above collapse and functionally behave the same.

\subsection{Different maximum force for the two contact models}

In the earlier subsections, results show that for a given maximum force the steady-state cohesion for the two contact models functionally behave the same under equal force and equal energy conditions. To study the functional form for the two models under different maximum force conditions, we compare the macroscopic behavior of the linear model to the liquid bridge model results for different surface tensions. Linear model simulations equivalent to surface tension 0.040 and $0.060 \mathrm{~N} \mathrm{~m}^{-1}$ are run with an equivalent adhesive stiffness 2 times and 3 times of that given by Eq. (29) keeping the interaction distance the same. Figure 19 shows a comparison

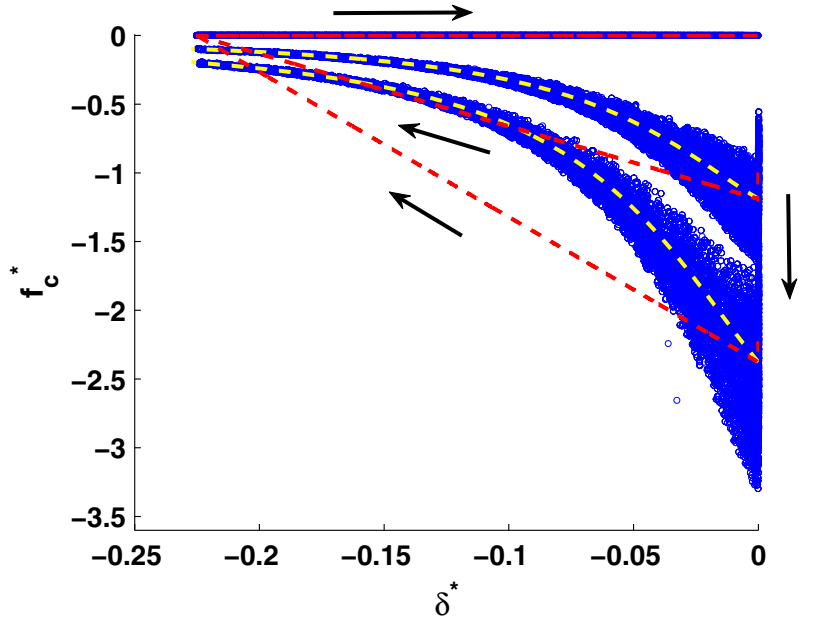

Fig. 19 Scaled adhesive force $f^{*}\left(f_{\mathrm{a}}{ }^{*}\right.$ for linear adhesive model and $f_{c}{ }^{*}$ for liquid bridge model) as a function of $\delta^{*}$ for the linear irreversible contact model (red), compared with the liquid bridge model (blue) for different maximum force and equal interaction distance. The yellow lines represent the force for the liquid bridge contact model for mean particle diameter $d_{\mathrm{p}}$ as a function of $\delta^{*}$. The arrow shows the loading and the unloading directions for the short-range forces. (Color figure online)

of the force-overlap for the two contact models for surface tension of liquid 0.020 and $0.040 \mathrm{~N} \mathrm{~m}^{-1}$.

Figure 20a shows the dependence of steady-state cohesion on the adhesive energy dissipated by the particles per contact for different $f_{\mathrm{a}}{ }^{\max }$ for the liquid bridge model and the linear model. For the same energy dissipated per contact, a higher surface tension of the liquid results in a higher macroscopic cohesion. Figure $20 \mathrm{~b}$ shows that $c^{*} / \gamma^{*}$ is a function of $E^{*} / \gamma^{*}$ for a given surface tension, or maximum force.

\section{Conclusion}

We observed a correlation between the steady-state cohesion and the microscopic parameters of the liquid bridge model. The microparameters are the liquid bridge volume, the liquid surface tension, the contact angle (which was kept constant), and the size of particles (i.e., curvature, which was also not varied). A detailed study of the effect of liquid bridge volume and surface tension of the liquid was done in this paper. These microscopic parameters control the macroscopic cohesion in wet granular materials in different ways. The steady-state cohesion of the system is proportional to the maximum adhesive force, which varies linearly with the surface tension. On the other hand, the steady-state cohesion is also linearly dependent on the maximum interaction distance between the particles, which depends on the volume of the liquid bridge. From these results, we have obtained a good micro-macro 

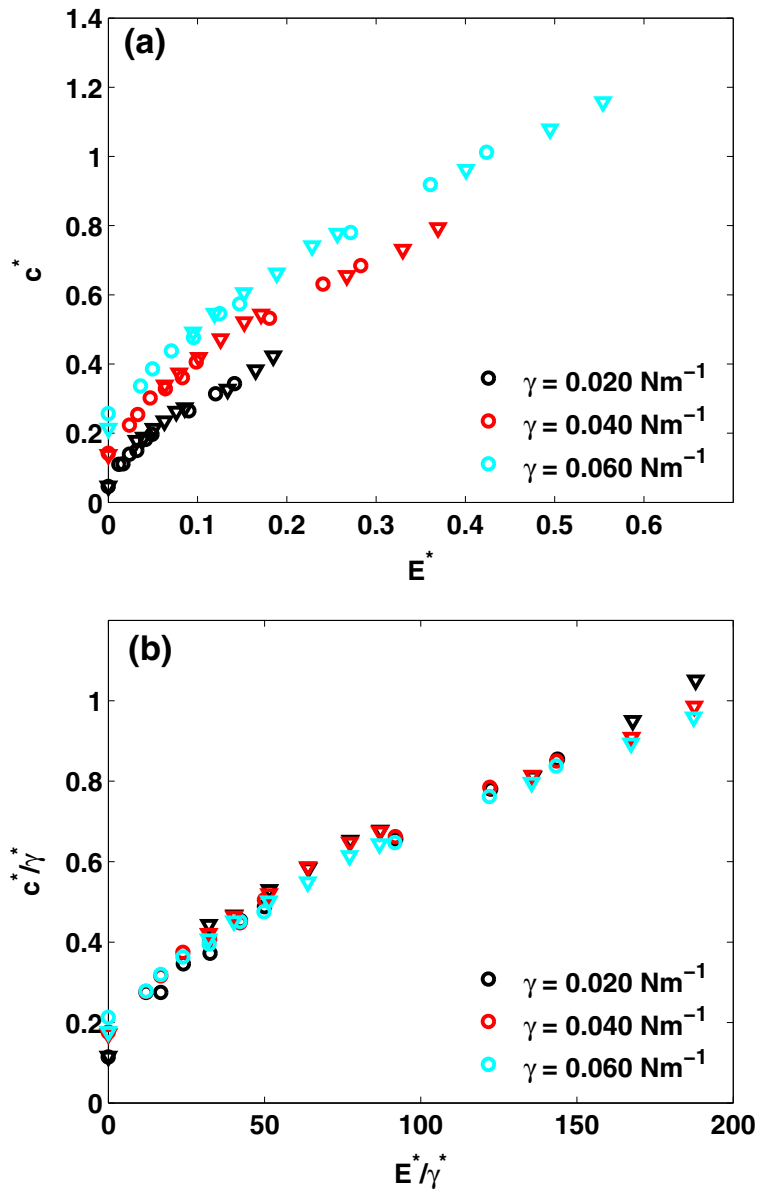

Fig. 20 a $c^{*}$ as a function of $E^{*}$ for different surface tensions of liquid. b $\frac{c^{*}}{\gamma^{*}}$ as a function of $\frac{E^{*}}{\gamma^{*}}$ for a different surface tensions of liquid as compared with the linear irreversible model. Different symbols denote $\circ$ liquid bridge model and $\nabla$ linear irreversible model

correlation between the steady-state cohesion and the microscopic parameters studied.

We analyzed the effect of cohesion on the wall torque required to rotate the system at a given rate. The torque (experimentally accessible) and the steady-state cohesion of the system are proportional and show similar linear dependence on the microscopic parameters.

Finally, an analogy was established between the liquid bridge model and a simpler linear irreversible contact model; even though these two models have different micro-macro correlations, the steady-state cohesion for the two models is the same if the maximum force and the total adhesive energy dissipated per contact for the two models are matched, irrespective of the shape of the attractive force function acting between the particles. In this way one can always replace a non-linear liquid bridge force by a simpler, faster to compute, linear one, and obtain identical macroscopic properties in less computational time. Furthermore, results for the two types of contact models with equal energy and different magnitudes of the maximum force show that they have different steady-state cohesions. The adhesive energy is thus not the sole microscopic condition for the two contact models to have same steady-state cohesion. Instead, both adhesive energy and cohesion scale linearly with the maximum adhesive force. The scaled cohesion for the two contact models is same for equal scaled adhesive energy. In this way, we can determine the steady-state cohesion from the two microscopic parameters, the adhesive energy and the maximum force.

In this paper, our study was focused on the micromacro correlations and comparing different contact models. It would be interesting to study the forces and their probability distributions for wet cohesive systems [1]. Future studies will aim at understanding the microscopic origin and dynamics of the contacts and liquid bridges throughout the force network(s) and also the directional statistics of the inter-particle forces inside a shear band. The effect of liquid migration on the macroproperties and a continuum description for wet, sheared granular materials will be studied in the near future.

Acknowledgments We acknowledge our financial support through STW Project 12272 "Hydrodynamic theory of wet particle systems: Modeling, simulation and validation based on microscopic and macroscopic description."

Open Access This article is distributed under the terms of the Creative Commons Attribution 4.0 International License (http://creativecomm ons.org/licenses/by/4.0/), which permits unrestricted use, distribution, and reproduction in any medium, provided you give appropriate credit to the original author(s) and the source, provide a link to the Creative Commons license, and indicate if changes were made.

\section{References}

1. Singh A, Magnanimo V, Saitoh K, Luding S (2014) Effect of cohesion on shear banding in quasistatic granular materials. Phys Rev E 90(2):022202

2. Mitarai N, Nakanishi H (2009) Simple model for wet granular materials with liquid clusters. Europhys Lett 88(6):64001

3. Ulrich S, Aspelmeier T, Roeller K, Fingerle A, Herminghaus S, Zippelius A (2009) Cooling and aggregation in wet granulates. Phys Rev Lett 102(14):148002

4. Strauch S, Herminghaus S (2012) Wet granular matter: a truly complex fluid. Soft Matter 8(32):8271-8280

5. Huang N, Ovarlez G, Bertrand F, Rodts S, Coussot P, Bonn D (2005) Flow of wet granular materials. Phys Rev Lett 94(2):028301

6. Huang N, Bonn D (2007) Viscosity of a dense suspension in Couette flow. J Fluid Mech 590:497-507

7. Bonnoit C, Lanuza J, Lindner A, Clement E (2010) Mesoscopic length scale controls the rheology of dense suspensions. Phys Rev Lett 105(10): 108302

8. Weinhart T, Thornton AR, Luding S, Bokhove O (2012) From discrete particles to continuum fields near a boundary. Granul Matter 14(2):289-294 
9. Thornton A, Weinhart T, Luding S, Bokhove O (2012) Modeling of particle size segregation: calibration using the discrete particle method. Int J Mod Phys C 23(08):1240014

10. Schall P, van Hecke M (2009) Shear bands in matter with granularity. Ann Rev Fluid Mech 42(1):67

11. Howell D, Behringer R, Veje C (1999) Stress fluctuations in a 2D granular Couette experiment: a continuous transition. Phys Rev Lett 82(26):5241

12. Schwarze R, Gladkyy A, Uhlig F, Luding S (2013) Rheology of weakly wetted granular materials: a comparison of experimental and numerical data. Granul Matter 15(4):455-465

13. Gladkyy A, Schwarze R (2014) Comparison of different capillary bridge models for application in the discrete element method. Granul Matter 16(6):911-920

14. Herminghaus S (2005) Dynamics of wet granular matter. Adv Phys 54(3):221-261

15. Richefeu V, El Youssoufi MS, Radjaï F (2007) Shear strength of unsaturated soils: experiments, DEM simulations, and micromechanical analysis. In: Theoretical and numerical unsaturated soil mechanics. Springer, pp 83-91

16. Darabi P, Li T, Pougatch K, Salcudean M, Grecov D (2010) Modeling the evolution and rupture of stretching pendular liquid bridges. Chem Eng Sci 65(15):4472-4483

17. Mani R, Kadau D, Or D, Herrmann HJ (2012) Fluid depletion in shear bands. Phys Rev Lett 109(24):248001

18. Mani R, Kadau D, Herrmann HJ (2013) Liquid migration in sheared unsaturated granular media. Granul Matter 15(4):447-454

19. Willett CD, Adams MJ, Johnson SA, Seville JP (2000) Capillary bridges between two spherical bodies. Langmuir 16(24):93969405

20. Alencar AM, Wolfe E, Buldyrev SV (2006) Monte Carlo simulation of liquid bridge rupture: application to lung physiology. Phys Rev E 74(2):026311

21. Lubarda VA (2015) On the stability of a cylindrical liquid bridge. Acta Mech 226(2):233-247

22. Soulie F, Cherblanc F, El Youssoufi MS, Saix C (2006) Influence of liquid bridges on the mechanical behaviour of polydisperse granular materials. Int J Numer Anal Methods Geomech 30(3):213-228

23. Singh A, Magnanimo V, Luding S (2015) A contact model for sticking of adhesive mesoscopic particles. arXiv preprint arXiv:1503.03720

24. Lätzel M, Luding S, Herrmann HJ (2000) Macroscopic material properties from quasi-static, microscopic simulations of a twodimensional shear-cell. Granul Matter 2(3):123-135

25. Wang X, Zhu H, Yu A (2012) Microdynamic analysis of solid flow in a shear cell. Granul Matter 14(3):411-421

26. Woldhuis E, Tighe BP, van Saarloos W (2009) Wide shear zones and the spot model: implications from the split-bottom geometry. Eur Phys J E 28(1):73-78

27. Roy S, Luding S, Weinhart T (2015) Towards hydrodynamic simulations of wet particle systems. Proc Eng 102:1531-1538
28. Singh A, Magnanimo V, Saitoh K, Luding S (2015) Role of gravity or pressure and contact stiffness in granular rheology. New J Phys 17:043028

29. Scheel M, Seemann R, Brinkmann M, Di Michiel M, Sheppard A, Breidenbach B, Herminghaus S (2008) Morphological clues to wet granular pile stability. Nat Mater 7(3):189-193

30. Mitarai N, Nori F (2006) Wet granular materials. Adv Phys 55(12): $1-45$

31. Denoth A (1982) The pendular-funicular liquid transition and snow metamorphism. J Glaciol 28(99):357-364

32. Weigert T, Ripperger S (1999) Calculation of the liquid bridge volume and bulk saturation from the half-filling angle. Part Part Syst Char 16(5):238-242

33. Pietsch W, Rumpf H (1967) FluÈssigkeitsvolumen und Grenzwinkel einer FluËssigkeitsbruÈcke zwischen zwei Kugeln. Chemie Ingenieur Technik 39:885

34. Schubert H (1982) Kapillarität in porösen Feststoffsystemen. Springer, Berlin

35. Erle MA, Dyson D, Morrow NR (1971) Liquid bridges between cylinders, in a torus, and between spheres. AIChE J 17(1):115-121

36. Deryaguin B (1934) Untersuchungen über die reibung und adhäsion, IV, Theorie des Anhaftens kleiner teilchen, Untersuchungen über die reibung und adhäsion, iv, theorie des anhaftens kleiner teilchen

37. Lian G, Thornton C, Adams MJ (1993) A theoretical study of the liquid bridge forces between two rigid spherical bodies. J Colloid Interface Sci 161(1):138-147

38. Rabinovich YI, Esayanur MS, Moudgil BM(2005) Capillary forces between two spheres with a fixed volume liquid bridge: theory and experiment. Langmuir 21(24):10992-10997

39. Willett C, Adams M, Johnson S, Seville J (2003) Effects of wetting hysteresis on pendular liquid bridges between rigid spheres. Powder Technol 130(1):63-69

40. Luding S (2008) The effect of friction on wide shear bands. Part Sci Technol 26(1):33-42

41. Luding S (2008) Constitutive relations for the shear band evolution in granular matter under large strain. Particuology 6(6):501-505

42. Luding S, Alonso-Marroquín F (2011) The critical-state yield stress (termination locus) of adhesive powders from a single numerical experiment. Granul Matter 13(2):109-119

43. Bouwman A, Henstra M, Hegge J, Zhang Z, Ingram A, Seville J, Frijlink H (2005) The relation between granule size, granule stickiness, and torque in the high-shear granulation process. Pharmaceut Res 22(2):270-275

44. Obraniak A, Gluba T (2012) Model of energy consumption in the range of nucleation and granule growth in drum granulation of bentonite. Physicochem Probl Miner Process 48(1):121-128

45. Wortel GH, Dijksman JA, van Hecke M (2014) Rheology of weakly vibrated granular media. Phys Rev E 89(1):012202 